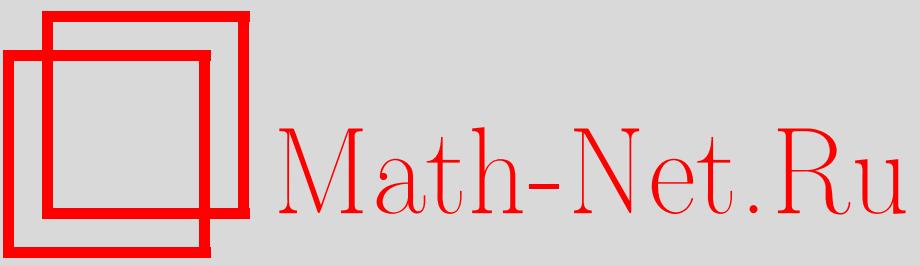

О. А. Чалых, Дуальность обобщенных задач Калоджеро и Рудженаарса, УМH, 1997, том 52, выпуск 6, 191-192

DOI: https://doi.org/10.4213/rm917

Использование Общероссийского математического портала Math-Net.Ru подразумевает, что вы прочитали и согласны с пользовательским соглашением

http://www.mathnet.ru/rus/agreement

Параметры загрузки:

IP : 3.85 .7 .115

26 апреля 2023 г., 15:16:58 


\title{
ДУАЛЬНОСТЬ ОБОБШЕННЫХ ЗАДАЧ КАЛОДЖЕРО И РУДЖЕНААРСА
}

\author{
О. А. ЧАлых
}

Рассмотрим квантовую задачу Калоджеро-Мозера-Сазерленда [1], [2], соответствующий оператор Шрёдингера в $\mathbb{R}^{n}$ имеет вид

$$
\mathscr{H}=-\Delta+\sum_{i<j} \frac{1}{2} m(m+1) \omega^{2} \operatorname{sh}^{-2} \frac{\omega}{2}\left(x_{i}-x_{j}\right),
$$

где $\Delta$ - оператор Лапласа в $\mathbb{R}^{n}, m$ и $\omega$ - произвольные параметры. В работе [3] А.П. Веселовым, К.Л. Стыркасом и автором было показано, что для натуральных значений $m$ оператор (1) обладает собственной функцией $\psi(k, x)\left(k \in \mathbb{R}^{n}\right.$ - спектральньй параметр) вида $\psi=P(k, x) \exp \left(k_{1} x_{1}+\cdots+k_{n} x_{n}\right)$, где $P$ - многочлен по $k$. При этом функция $\psi$ однозначно определяется следующими аналитическими свойствами:

(А) старшие члены многочлена $P$ имеют вид $\prod_{i<j}\left(k_{i}-k_{j}\right)^{m}$;

(Б) для каждого вектора $\alpha \in \mathbb{R}^{n}$ вида $\alpha=\frac{\omega}{2}\left(e_{i}-e_{j}\right)$ и $s=1, \ldots, m$

$$
\psi(k-s \alpha, x)=\psi(k+s \alpha, x) \text { при }(\alpha, k)=0 .
$$

Наличие такой $\psi$-функции, которую естественно назьвать многомерной функцией Бейкера-Ахиезера в соответствии с традицией, идущей от работ И.М. Кричевера [4], позволяет построить коммутативное кольцо дифференциальных операторов, содержащее оператор (1) и изоморфное кольцу $\mathscr{R}$ многочленов $f(k)$, обладающих свойством (Б): $f(k-s \alpha)=f(k+s \alpha)$ при $(\alpha, k)=0$ для всех $s=1, \ldots, m$ (см. [3]). При этом $\psi$ является общей собственной функцией этого кольца.

Оказьвается, $\psi(k, x)$ как функция спектрального параметра является общей собственной функцией коммутативного кольца разностныл Рудженаарса [5]. Система Рудженаарса, являющаяся разностным аналогом системы Калоджеро, образована $n$ коммутируюшими операторами $D_{1}, \ldots, D_{n}$, простейший из которых $D=D_{1}$ имеет вид

$$
D=\sum_{i=1}^{n} \prod_{j \neq i}^{n}\left(1-\frac{m \omega}{k_{i}-k_{j}}\right) T_{i}^{\omega},
$$

где $T_{i}^{\omega}$ - оператор сдвига, действующий на функцию $f(k)$ по формуле $\left(T_{i}^{\omega} f\right)\left(k_{1}, \ldots, k_{i}, \ldots, k_{n}\right)$ $=f\left(k_{1}, \ldots, k_{i}+\omega, \ldots, k_{n}\right)$. (Формулы для $D_{2}, \ldots, D_{n}$ для тригонометрического случая см. в работе [6], рациональньй предел отвечает $\alpha \rightarrow 0$.)

Теорема 1. Многомерная функиия Бейкера-Ахиезера $\psi(k, x)$, определяемая условиями (А)-(Б), является собственной для рациональных операторов Рудженаарса $D_{1}, \ldots, D_{n} . \Pi p u$ әтом $D_{i} \psi=\lambda_{i}(x) \psi$, где $\lambda_{i}(x)$ есть $i$-я әлементарная симметрическая функция от $e^{\omega x_{1}}, \ldots, e^{\omega x_{n}}$.

Таким образом, рациональная система Рудженаарса является биспектрально дуальной в смысле Дюйстермата-Грюнбаума [7] для тригонометрической задачи Калоджеро. Отметим, что, как нетрудно вьвести из теоремы 1 , такая дуальность имеет место для произвольного (не обязательно целого) $m$ в том смысле, что собственная функция задачи (1) при подходящей нормировке является собственной для задачи (2). В простейшем случае $n=2$ это было отмечено Рудженаарсом [8], им же высказывалось предположение, что подобное утверждение справедливо для всех $n$.

Рассмотрим теперь деформацию квантовой задачи Калоджеро-Мозера, предложенную в работе [9], соответствующий оператор Шрёдингера в $\mathbb{R}^{n+1}$ имеет вид

$$
\widehat{\mathscr{H}}=\mathscr{H}-\frac{\partial^{2}}{\partial x_{n+1}^{2}}+\sum_{i=1}^{n} \frac{(m+1)}{2} \omega^{2} \operatorname{sh}^{-2} \frac{\omega}{2}\left(x_{i}-\sqrt{m} x_{n+1}\right),
$$

где $\mathscr{H}$ - оператор (1), $m$ и $\omega$-произвольные параметры. Как отмечено в работе [9], оператор (3) является интегрируемым при всех $m$ и алгебраически интегрируемым при целшіх $m$. (Заметим,

Работа вьполнена при частичной поддержке Российского фонда фундаментальных исследований (грант № 97-01-01404) и INTAS (грант № 93-166-ext). 
что при $m=1$ задача (3) сводится к специальному случаю задачи (1).) Соответствующая цельм значениям $m$ функция Бейкера-Ахиезера имеет вид $\psi=P(k, x) \exp \left(k_{1} x_{1}+\cdots+k_{n+1} x_{n+1}\right)$, $P$ - многочлен по $k$, и однозначно определена следующими аналитическими свойствами:

(A1) старшие члены многочлена $P$ имеют вид $\prod_{i<j}^{n}\left(k_{i}-k_{j}\right)^{m} \prod_{i=1}^{n}\left(k_{i}-\sqrt{m} k_{n+1}\right)$;

(Б1) для $\alpha=\frac{\omega}{2}\left(e_{i}-\sqrt{m} e_{n+1}\right)$ выполняется $\psi(k-\alpha, x)=\psi(k+\alpha, x)$ при $(\alpha, k)=0$, а для $\alpha=\frac{\omega}{2}\left(e_{i}-e_{j}\right), 1 \leqslant i<j \leqslant n$, вьполнены условия (Б).

Как и в случае задачи (1), $\psi$ является общей собственной функцией коммутативного кольца дифференциальных операторов (по $x$ ), содержащего оператор (3) и изоморфного колшцу $\widehat{\mathscr{R}}$ многочленов $f(k)$, удовлетворяющих условиям (Б1). Чтобы описать дуальную систему разностных операторов по $k$, рассмотрим следующую деформацию оператора Рудженаарса (2): (4)

$$
\widehat{D}=\sum_{i=1}^{n}\left(1-\frac{\omega}{k_{i}-\sqrt{m} k_{n+1}+\gamma}\right) \prod_{j \neq i}^{n}\left(1-\frac{m \omega}{k_{i}-k_{j}}\right) T_{i}^{\omega}+m^{-1} \prod_{i=1}^{n}\left(1+\frac{m \omega}{k_{i}-\sqrt{m} k_{n+1}+\gamma}\right) T_{n+1}^{\sqrt{m} \omega} .
$$

Здесь $T_{i}^{\omega}$ имеют тот же смысл, что и в формуле $(2), \gamma=(1-m) \omega / 2$. Введем также следующие многочлены $f_{j}=\left(k_{1}\right)^{j}+\cdots+\left(k_{n}\right)^{j}+p_{j}\left(k_{n+1}\right)$, где $p_{j}(t)$ однозначно определяется для всех $m \neq 0$ и $j=1,2, \ldots$ из тождества $p_{j}(t+\sqrt{m} \omega)-p_{j}(t)=(\sqrt{m} t+(m+1) \omega / 2)^{j}-$ $(\sqrt{m} t+(m-1) \omega / 2)^{j}$ и условия $p_{j}(0)=0$. Смысл тождества в том, что оно обеспечивает вьполнение условий (Б1) для многочлена $f_{j}$.

Рассмотрим разностные операторы $\widehat{D}_{j}=\frac{1}{j ! \omega^{j}}\left(\operatorname{ad}_{\widehat{D}}\right)^{j}\left[f_{j}\right], j=2, \ldots, n+1$, где $\widehat{D}$ - оператор (4), $f_{j}$ понимается как оператор умножения на функцию, а $\left(a d_{\widehat{D}}\right)^{j}$ означает многократное применение операции ad: $\operatorname{ad}_{A} B=A B-B A$.

Tеорема 2. Разностные операторы $\widehat{D}_{1}=\widehat{D}, \widehat{D}_{2}, \ldots, \widehat{D}_{n+1}$ коммутируют для любого $m$. Для $m \in \mathbb{Z}_{+}$функиия Бейкера-Ахиезера $\psi(k, x)$ задачи (3), определяемая условиями (A1)-(Б1), является собственной для операторов $\widehat{D}_{1}, \ldots, \widehat{D}_{n+1}$. При этом $\widehat{D}_{j} \psi=\lambda_{j}(x) \psi$, где $\lambda_{j}(x)=e^{j \omega x_{1}}+\cdots+e^{j \omega x_{n}}+\frac{1}{m} e^{j \sqrt{m} \omega x_{n+1}}$.

ЗАмечАния. 1. Из теоремы 2 так же, как и для задачи (1), можно заключить, что системы (3) и (4) дуальны для произвольного (не обязательно целого) $m$.

2. Можно показать, что операторы (2) и (4) являются алгебраически интегрируемыми при целых $m$ в смысле [3].

3. С помощью оператора (2) можно указать следующую явную формулу для $\psi$-функции задачи Калоджеро (1): $\psi(k, x)=C(x)(D-\lambda)^{M}[A(k) \exp (k, x)]$, где $\lambda=e^{\omega x_{1}}+\cdots+e^{\omega x_{n}}$, оператор $(D-\lambda)^{M}$ применяется к выражению в квадратных скобках, $M=m n(n-1) / 2, A(k)$ - многочлен вида $\prod_{i<j} \prod_{s=1}^{m}\left[\left(k_{i}-k_{j}\right)^{2}-s^{2} \omega^{2}\right], C^{-1}(x)=M ! \prod_{i<j}\left(e^{\omega x_{i}}-e^{\omega x_{j}}\right)^{m}$. Аналогичная формула справедлива и для задачи (3). Эти формулы являются разностным аналогом формул, полученных Ю.Ю. Берестом в случае $\omega=0$.

Большая часть данной работы была выполнена во время моего пребьвания в университете Paris-XII (IUT, Fontainebleau) в июне 1997 г. Я глубоко признателен моим коллегам за гостеприимство. Я также хотел бы поблагодарить А. П. Веселова и Ю. Ю. Береста за чрезвычайно полезные обсуждения и советы.

\section{СПИСОК ЛИТЕРАТУРЫ}

[1] Calogero F. // J. Math. Phys. 1971. V. 12. P. 419-436. [2] Sutherland B. // Phys. Rev. A (5). 1972. Р. 1372-1376. [3] Веселов А. П., Стыркас К. Л., Чалых О. А. // ТМФ. 1993. Т. 94. №2. С. 253-275. [4] Кричевер И. М. // УМН. 1977. Т. 32. №6. C. 198-245. [5] Ruijsenaars S. N. M. // Comm. Math. Phys. 1987. V. 110. P. 191-213. [6] van Diejen J.F. // Compositio Math. 1995. V. 95. P. 183-233. [7] Duistermaat J., Grunbaüm F. A. // Comm. Math. Phys. 1986. V. 103. P. 177-240. [8] Ruijsenaars S. N. M. // Integrable and superintegrable systems / ed. B. Kupershmidt. Singapore: World Scientific, 1990. Р. 165-206. [9] Веселов А.П., Фейгин М. В., Чалых О.А. // УМН. 1996. Т. 51. №3. C. $185-186$. 